
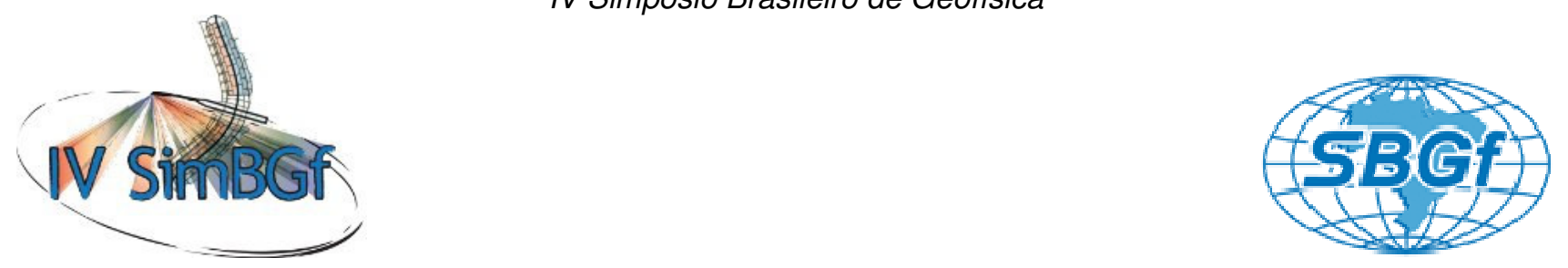

\title{
Modelagem 3D da Anomalia Magnética de Pratinha I (MG)
}

Vinicius Hector Abud Louro*, Instituto de Astronomia, Geofísica e Ciências Atmosféricas - Universidade de São Paulo Marta Silvia Maria Mantovani, Instituto de Astronomia, Geofísica e Ciências Atmosféricas - Universidade de São Paulo

\section{Copyright 2010, SBGf - Sociedade Brasileira de Geofísica}

Este texto foi preparado para a apresentação no IV Simpósio Brasileiro de Geofísica, Brasília, 14 a 17 de novembro de 2010. Seu conteúdo foi revisado pelo Comitê Técnico do IV SimBGf, mas não necessariamente representa a opinião da SBGf ou de seus associados. É proibida a reprodução total ou parcial deste material para propósitos comerciais sem prévia autorização da SBGf.

\section{Abstract}

The region of Pratinha I centered at coordinates (1945'S, 4610'W) - in the Alto do Paranaíba Province - presents a tripolar anomaly of about $50 \mathrm{~km}$ wide in its longer axis and high intensity. The aim of this study is the tridimensional modelling of the anomaly's source-body through aeromagnetometry data to estimate and suggest its nature, once it doesn't indicate any signs of outcrops.

\section{Introdução}

A CODEMIG (Companhia de Desenvolvimento Econômico de Minas Gerais) em 2006 realizou um aerolevantamento magnético e gamaespectrométrico sobre a região do sudoeste do Estado de Minas Gerais (Área 7). Neste, observa-se uma anomalia magnética centrada nas coordenadas (1945'S, 4610'W, Figura 1) de característica tripolar e grande contraste de susceptibilidade. Esta anomalia foi denominada Pratinha I e não apresenta sinais de afloramento. Está contida na Faixa de Dobramento Brasília, mais especificamente no grupo Canastra Indiviso, de origem no período Mesoproterozóico Esteniano e geologia caracterizada por metarenitos, quartizitos puros e micáceos, grafitas xisto, sericitas xisto, quartzo-muscovitas xisto, filitos, grafitas filito, cloritas filito, metassiltitos, metargilitos siltíticos, margas, calcáreos e ardósias. Segundo Rodrigues (2008), estudos de Sm-Nd e U-Pb sugerem a idade deste grupo inferior a $1 \mathrm{Ga}$.

A região caracteriza-se pela exploração de minerais como fosfatos, ouro, nióbio, entre outros, e se localiza próxima a outros pólos de exploração mineral como Tapira e Araxá.

Uma vez que não existem indícios de afloramento que possam ser relacionados à anomalia recorre-se a métodos geofísicos indiretos para a inferência da natureza da fonte da anomalia. Para o caso em estudo foi utilizado o método potencial magnético a partir dos dados do aerolevantamento da CODEMIG, os quais foram processados e interpretados com 0 intuito de identificar as características da estrutura em questão elaborando modelos representativos do corpo a partir de seu contraste de susceptibilidade magnética com a rocha encaixante.

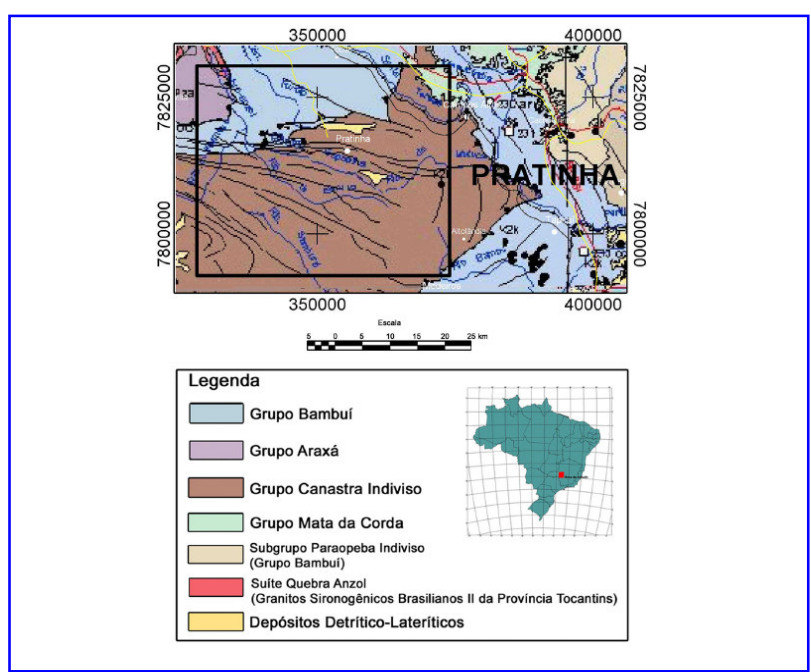

Figura 1-Mapa geológico da região da anomalia de Pratinha I (Adaptado da Carta Geológica do Brasil ao Milionésimo, Folha SE.23, Belo Horizonte)

\section{Metodologia}

A magnetização de uma rocha é dada pela soma vetorial das magnetizações induzida e remanente, definidas respectivamente pela susceptibilidade e pela remanência magnética do material.

A modelagem magnética 3D proposta neste estudo é realizada a partir dos contrastes de susceptibilidade entre estruturas-fonte e rocha-encaixante.

Para a interpretação da anomalia magnética, inicialmente foi removida a influência do campo magnético regional (trend). O processo de remoção de trend por filtro polinomial ocorre pela expansão das células do grid original em no mínimo a $10 \%$ e aumentando até que atinja uma área máxima aceitável de acordo com a ordem definida para o trend por meio do cálculo da FFT de ordem 2. Outro filtro utilizado é o gaussiano que suaviza o "grid" aplicando a FFT de ordem 1.

A partir do resultado dessa remoção foram compostos mapas de sinal analítico, redução ao pólo, tanto por meio da redução proposta por Baranov (1957), quanto pelo algoritmo proposto por Fedi et al. (1994), Integral Vertical do Sinal Analítico (VIAS), Sinal Analítico da Integral Vertical (ASVI) e Amplitude do Campo Magnético Anômalo.

O sinal analítico de uma anomalia magnética dará origem a um mapa contendo o módulo do vetor de 
magnetização, seja da remanente quanto da induzida, Este procedimento é utilizado principalmente para a delimitação das bordas do corpo investigado.

O método de redução ao pólo (RTP) se dá pela simulação do comportamento da anomalia investigada como se a mesma se localizasse no pólo magnético da Terra. Este procedimento é realizado para a identificação da amplitude do sinal do corpo anômalo e de seus limites. Dois algoritmos diferentes foram utilizados para esta redução com fins comparativos e serão abordados na discussão deste trabalho.

Tanto o VIAS quanto o ASVI são métodos de contorno para a inversão $3 \mathrm{D}$ na presença de magnetização remanente (Paine et al. 2001). Da mesma forma é utilizado o algoritmo da Amplitude do Campo Magnético Anômalo referido por Shearer (2005).

Todos os grids gerados foram invertidos com o auxílio dos códigos de inversão desenvolvidos por $\mathrm{Li}$ e Oldenburg (1996).

\section{Resultados}

O trabalho de remoção de trend envolvendo subtração de grids, filtros polinomiais de ordens 1 a 9 e gaussianos com comprimentos de onda de 15 a $70 \mathrm{~km}$, indicou que o filtro polinômial de ordem 1 foi o que isolou mais satisfatoriamente a anomalia do campo magnético regional (Figura 2).

Do grid resultante foram compostos mapas de sinal analítico, RTP, VIAS e ASVI, com componentes sugeridas pelo IGRF de inclinação $-29.71^{\circ}$, declinação $-20.51^{\circ}$ e intensidade $23270 n T$.

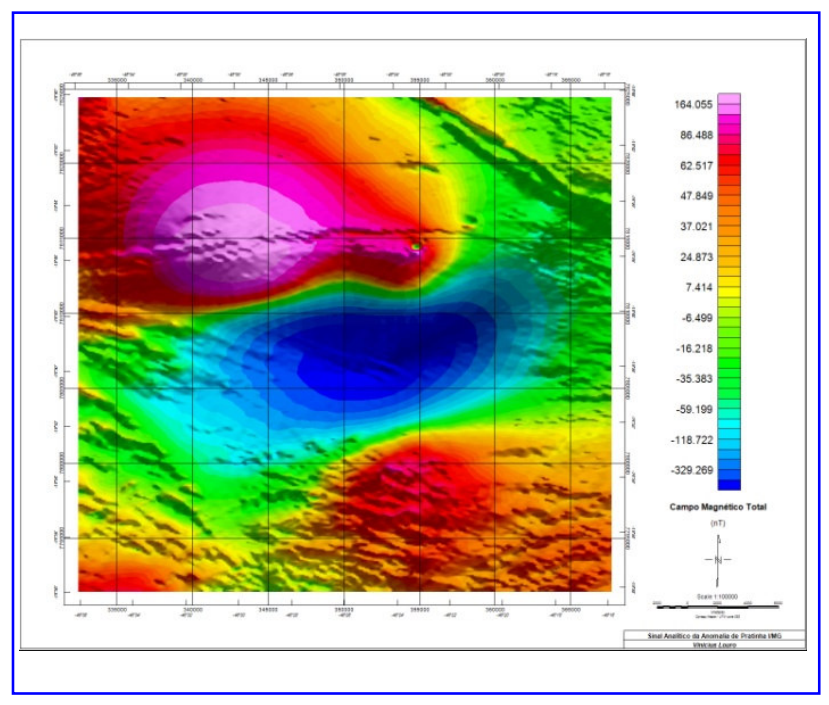

Figura 2 - Campo Magnético Total com Trend de Ordem 1 removido

As reduções ao pólo foram realizadas por meio de dois algoritmos: um proposto por Baranov (1957) e outro por Fedi et al. (1994).
A RTP proposta por Baranov (1957) consiste na redução da anomalia ao pólo magnético da Terra seguindo a função:

$$
L(\theta)=\frac{[\sin (D)-i \cos (D \cdot \cos (D-\theta)]}{\left[\sin ^{2}(0,2) \cos ^{2}(Q) \cos ^{2}(D-\theta)\right]\left[\operatorname { s i n } ^ { 2 } \left(D-\cos ^{2}\left[D \cos ^{2}(D-\theta)\right]\right.\right.}(1)
$$

Sendo I a inclinação, $D$ a declinação, la a correção da inclinação do campo magnético e $\theta 0$ azimute.

O algoritmo de Fedi et al. (1994) faz uma série de reduções com diversas inclinações (I) e declinações (D), até encontrar um par (I,D) que, converge dentro que um raio de tolerância e represente a resposta de um corpo situado no pólo magnético. $O$ conjunto obtido foi: Inclinação $25.6^{\circ}$ e Declinação $-35.2^{\circ}$.

Os métodos VIAS e ASVI geraram ambos altos magnéticos em relação ao campo regional, com um comportamento melhor definido pelo segundo. $O$ intervalo de amplitude do ASVI resultou na metade do valor apresentado pelo método VIAS.

A amplitude do campo anômalo gerou um resultado similar ao do método VIAS, com uma variação de cerca de 400nT (Figura 3).

Foram executadas diversas inversões entre as quais oito tiveram resultados significativos. Estes resultados podem ser divididos em dois grupos identificados por sua semelhança em forma, contraste de susceptibilidade e profundidade do topo do modelo.

O primeiro grupo de inversões é formado pelo campo total - invertendo o grid com a inserção da inclinação e declinação obtida pelo algoritmo de Fedi et al. (1994) -, redução ao pólo pelo mesmo algoritmo e VIAS. Neste grupo foram obtidas formas cilindricas ovaladas, com seu topo entre 1700 e 2000 metros de profundidade e contraste de susceptibilidade de cerca de $0.2(\mathrm{SI})$.

Já o segundo grupo composto pelas inversões ASVI e Amplitude do Campo Magnético Anômalo, apresentaram profundidades mais acentuadas de 2500 a 2700 metros e menor contraste de susceptibilidade, cerca de 0.13 (SI). É interessante notar que, apesar das diferenças de profundidade e susceptibilidade, a forma do corpo foi preservada. As inversões do campo magnético total sem a inserção dos dados sugeridos pelo algoritmo de Fedi et al. (2004) e a redução ao pólo método de Baranov (1957) apresentaram um corpo de forma diferente, assemelhando-se a um coração, uma profundidade de cerca de 1500 metros, mas ainda assim mantendo um contraste de $0.13(\mathrm{SI})$.

\section{Discussão e Conclusões}

Os resultados obtidos com 0 processamento e modelagens permitiram observar a existência de um corpo intrusivo de forma cilíndrica-ovalada. É possível notar, em comparação com o mapa geológico local que o eixo menor do cilindro tem direção N-S, mantendo a face norte do corpo paralela a uma falha de grande porte na direção E-W, e a face oeste acompanhando uma 
segunda falha de menor porte na direção NW-SE. Os modelos obtidos pelo campo magnético total sem atribuições e pela redução proposta por Baranov (1957) enfatizam essa tendência (Figura 3).

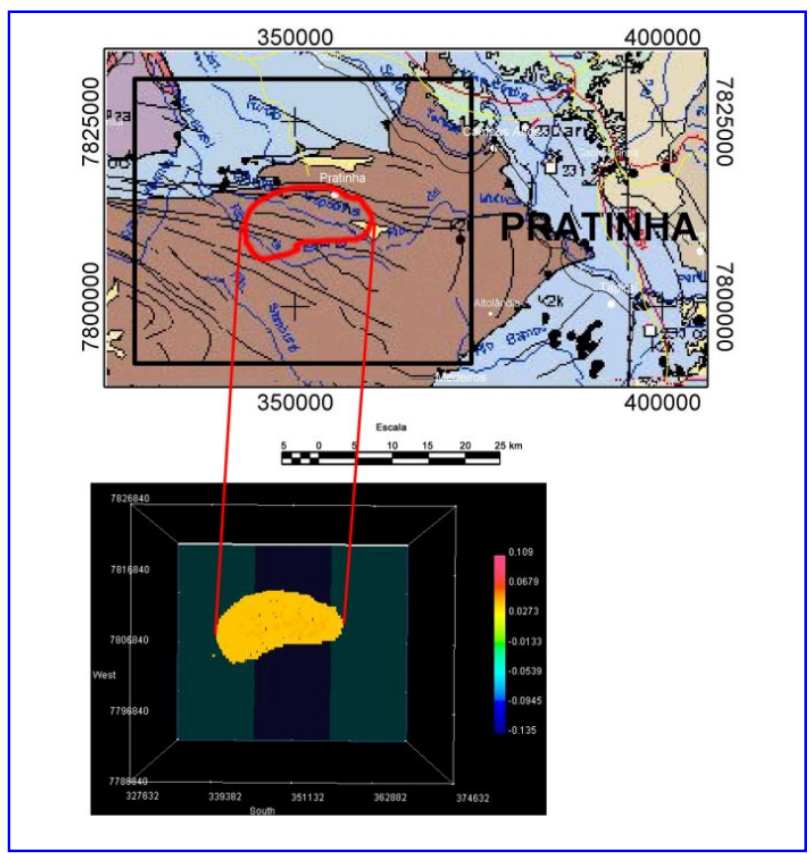

Figura 3 - Comparação entre a vista de topo do modelo obtido pela inversão do grid de Campo Magnético Total sem a inclinação e declinação sugerida pelo algoritmo de Fedi et al (1995) e o mapa geológico da região de Pratinha (MG). (Adaptado da Carta Geológica do Brasil ao Milionésimo, Folha SE.23, Belo Horizonte)

A semelhança entre modelos gerados, pode ser atribuída a uma remanecência de baixa intensidade, de modo que o aplicativo mag3D não apresentou problemas para a inversão dos grids (Figura 4). Para a confirmação desta possibilidade foram invertidos os grids de ASVI, VIAS e Amplitude do campo Magnético Anômalo. Segundo Paine et al. (2001), a inversão dos grids de ASVI e VIAS não gerariam resultados de boa qualidade caso não houvesse magnetização remanente, $\mathrm{O}$ mesmo foi feito para o terceiro caso proposto por Shearer (2005), com resultado esperado para uma inversão com pouca dependência da componente induzida do campo magnético sendo semelhante aos demais.

De acordo com Paine et al. (2001), nos casos ASVI e VIAS seria esperado um corpo de maior raio, uma vez que estes processos implicam numa convergência mais lenta ao pico da anomalia, assim como uma maior profundidade do topo do corpo devido ao ganho que ocorre às baixas frequências do grid quando se realiza a integral vertical.

$\mathrm{Na}$ região da anomalia existe uma predominância de quartizitos e grafita-micaxistos, o que sugere para a rocha encaixante uma susceptibilidade em torno de 0.01 a 0.05 (SI) segundo Telford et al. (1990). A partir dos dois casos obtidos pelas inversões, a anomalia pode apresentar de 0.14 a 0.25 (SI) que pode ser atribuída a uma formação alcalina, tendo em vista a susceptibilidade média e a geologia local.

\section{Agradecimentos}

À CODEMIG, ao Geophysical Inversion Facility da University of British Columbia, à Reitoria da Universidade de São Paulo e aos componentes do grupo de Geofísica da Litosfera do IAG-USP (Infra-estrutura: CNPq, FAPESP e IAG).

\section{Referências}

BARANOV, V., 1957. A new method for interpretation of aeromagnetic maps: pseudo-gravimetric anomalies. Geophysics, vol. 22, n. 2, p. 359-383.

CPRM - Serviço Geológico do Brasil, 2004, Carta Geológica do Brasil ao Milionésimo, Folha SE.23, Belo Horizonte. Programa Geologia do Brasil. Brasília, Brasil.

FEDI, M.F., FLORIO, G., RAPOLLA, A.G., 1994. A method to estimate the total magnetization direction from a distortion analysis of a magnetic-anomalies. Geophys, Prospect, vol. 42, n. 3, p. 261-274.

LI, Y; OLDENBURG, D.W. , 1996. 3D Inversion of magnetic data. Geophysics, vol. 61, p. 394-408.

PAINE, J., HAEDERLE, M., FILS, M., 2001. Using transformed TMI data to invert dor remanently magnetized bodies. Exploration Geophysics, no 31, p. 238-242.

RODRIGUES, J. R., 2008. Proveniência de sedimentos dos grupos Canastra, Ibiá, Vazante e Bambuí - Um estudo de zircões detríticos e Idades Modelo Sm-Nd. Tese de Doutoramento, UnB, DF, 128 p.

SHEARER, S.E., 2005. Three-dimensional inversion of magnetic data in presence of remanent magnetization. Dissertação (Mestrado em geofísica). Center for Gravity, Electrical e Magnetic Studies, Colorado School of Mines. $148 \mathrm{p}$.

TELFORD, W.M; GELDART, L.P; SHERIFF, R.E. Applied geophysics. 1990. Cambridge University Press, USA. Segunda edição, p.74 

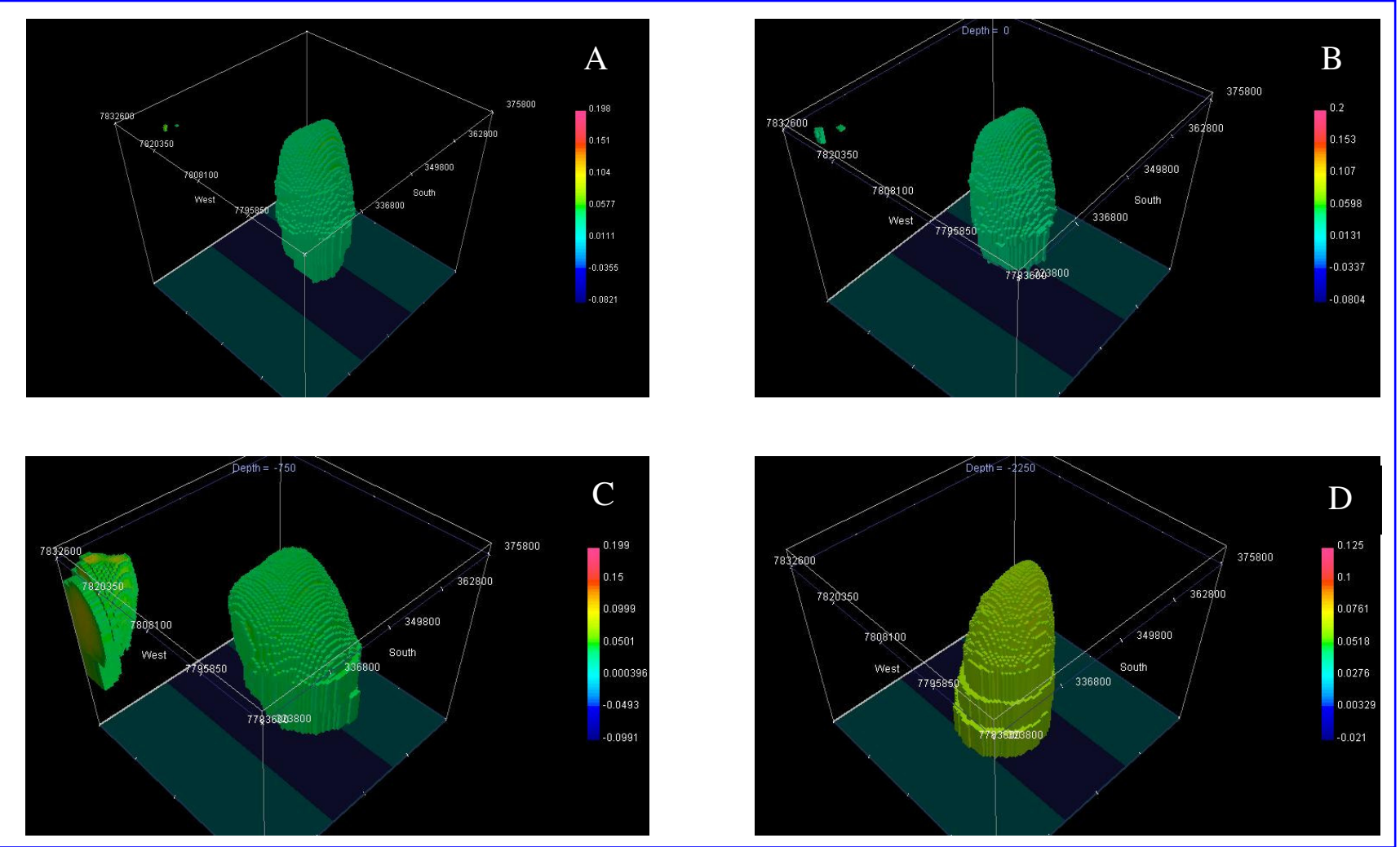

Figura 4 - Modelos obtidos pela inversão: (A) Campo Magnético Total com inclinaçãoe declinação sugeridas pelo algoritmo de Fedi et al (1994); (B) Redução ao Pólo com o mesmo algoritmo; (C) VIAS; (D) ASVI. 